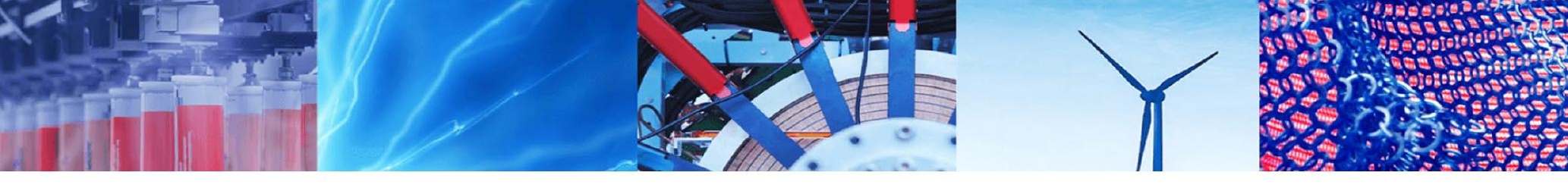

Research Article

\title{
A powerful model predictive control via stability condition for direct matrix converter
}

\author{
Minh-Duc Ngo ${ }^{1} \cdot$ Van-Quang-Binh $\mathrm{Ngo}^{2}$ (D) $\cdot$ Kim Anh Nguyen ${ }^{3} \cdot$ Dinh-Hieu Le ${ }^{4,5} \cdot H . \operatorname{Tran}^{2}$
}

Received: 8 April 2020 / Accepted: 6 November 2020 / Published online: 19 November 2020

(c) Springer Nature Switzerland AG 2020

\begin{abstract}
This paper offered a novel approach based on model predictive control for a direct matrix converter with a long prediction horizon. A discrete-time model of the dynamical system is used to predict the future response of the control variables for pursuing the reference load current. The long prediction horizon can be used to improve the control performance but increase the computational complexity. In contrast to the classical finite control set model predictive control, the proposed scheme employed a control Lyapunov function in the control design to guarantee a stable closed-loop system. During each sampling interval, only the probable control switching states which meet the sufficient stability condition are considered for evaluating the optimization processes, leading to a dramatic reduction of the computation time. An extensive examination of the conventional model predictive control and the proposed method under some operational circumstances is conducted with Matlab software. The simulation and experimental results prove the effectiveness and feasibility of the proposed control strategy in terms of stability and reduced computational burden.
\end{abstract}

Keywords Finite control set model predictive control · Direct matrix converter · Long prediction horizon · Calculation burden · Control Lyapunov function · Stability condition

\section{Introduction}

A direct matrix converter (DMC) provides many attractive features in comparison with the conventional two-stage conversion topology (back-to-back converter) such as without DC-link energy storage component, bidirectional power transfer and sinusoidal input and output currents as shown in Fig. 1 [1, 2]. Thus, the DMC becomes an alternative solution in many applications such as motor drive $[3,4]$, wind energy conversion system [5], and flexible AC transmission systems [6].

The first modulation method for matrix converter (MC) is called a direct transfer function which was proposed by Venturini [7]. In this technique, the output voltage is achieved by multiplying the modulation transfer matrix and input voltage. However, the major drawback of this method is the low voltage ratio transfer. Recently, pulse width modulation [8] and space vector modulation [9] have been developed for MC to obtain the complete regulation of both output voltage and input current, and improve the controller performance. Unfortunately, these methods have a low dynamic response and lack of controlled current source. This means that the performance of the system can be deteriorated if take into account the power factor control. In order to address these problems, several alternative approaches have been proposed for control $\mathrm{AC}$ machines using $\mathrm{MC}$ such as direct torque

$\triangle$ Van-Quang-Binh Ngo, nvqbinh@hueuni.edu.vn | ${ }^{2}$ Department of Automation, Thai Nguyen University of Technology, Thai Nguyen City 251750, Vietnam. ${ }^{2}$ Faculty of Physics, University of Education, Hue University, Thua Thien Hue 53000, Vietnam. ${ }^{3}$ The University of Danang - University of Science and Technology, 54 Nguyen Luong Bang, Danang, Vietnam. ${ }^{4}$ Information Technologies-Automated Control Systems, National University of Science and Technology MISiS, Moscow, Russia. ${ }^{5}$ Hue Industrial College, Thua Thien Hue 53000, Vietnam. 


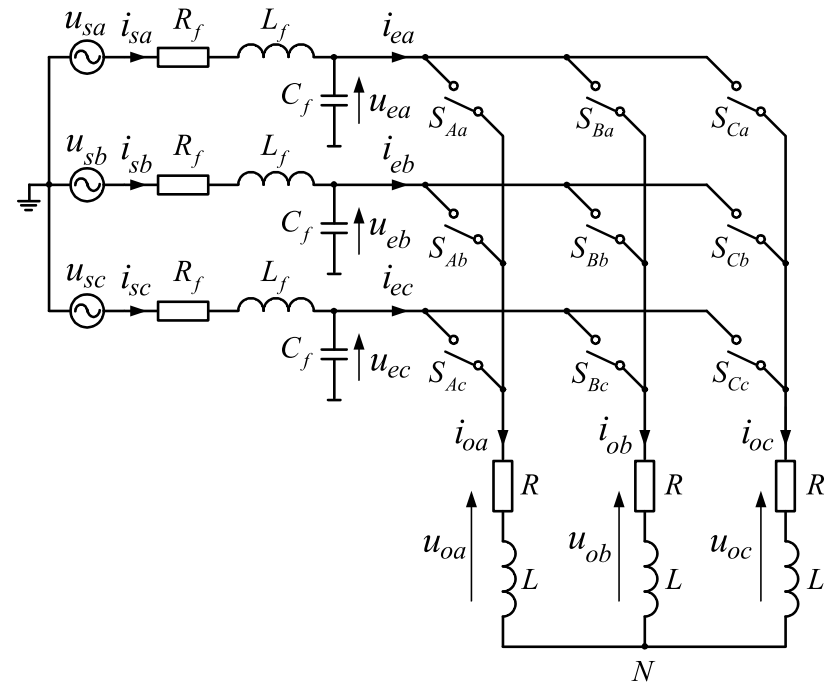

Fig. 1 The configuration of direct matrix converter

control $[3,5]$, sliding mode control $[10,11]$ and predictive control $[12,13]$.

In the last decade, model predictive control (MPC) has been attracted to control power electronics applications and electrical machines. In particular, the finite control set model predictive control (FCS-MPC) has been widely employed to DMC owing to its advantages in terms of easy implementation and simplicity of the control design [14-17]. Furthermore, the main benefit of FCS-MPC is that the non-linearities, additional constraints of the system and delay compensation can be taken into account in the controller. Despite this interest, a major drawback of the FCS-MPC implementation is a high computational burden. To obtain the optimal value, all switching states of the converters have to be calculated online for the enumeration of the cost function, leads to increase the computational time, especially with a high number of switching state and long prediction horizons. Therefore, this makes difficult for implementation with the standard digital hardware platform. In an attempt to solve this problem, an algorithm based on predictive control for DMC is proposed in [18] which decreases the calculation time by using preselected switching states based on sector distribution of the input current and output voltage vectors. Another method is introduced in [19] based on using the high performance of the field-programmable gate array to decrease the calculation time. In [20] a straightforward FCS-MPC for MC fed permanent magnet synchronous motor is employed to reduce the execution time of MPC algorithm. Another approach proposed in [21-24] used branch and bound strategies for other converters such as sphere decoder, alternating direction method of multipliers to reduce the computational requirement. However, these techniques need a modulation block which can increase the complexity of the control system. The limitation of their study is the implementation with powerful control platforms such as ARM processors and the Dspace system, leading to an increase in the cost of the system. Therefore, this is not suitable for real-time implementation with low-cost processors.

Studies into computational burden can be useful, but to counterbalance FCS-MPC for DMC, it is important to study the stability of the system. A useful approach to address this issue is the Lyapunov theorem on stability. Previous works have only concentrated on the application of Lyapunov function based MPC for two-level voltage source converter $[25,26]$. Their strategies are derived from the voltage reference vector in the objective function to decrease the execution time. Unfortunately, this approach is not well suited to a multilevel converter due to the evaluation of the optimization problem with all switching states. The present paper outlines a powerful approach based model predictive control for DMC by taking into account the computational burden and incorporating the stability in the control design. The predicted control variables of the input current are achieved by employing a discrete-time dynamic model of the DMC system. A long prediction horizon is employed to enhance the control performance of the system in terms of steady-state error and the total harmonic distortion (THD) of the current. However, this leads to a high computational cost as the large switching state that is an unexpected result. To overcome this obstacle, a control Lyapunov function is integrated into the loop optimization to ensure the stability of the closed-loop system. In our study, only available switching states of DMC which meet the stability condition are considered for optimizing the cost function. As a result, aside from the reinforcement of the stability, the computation time required of two-step prediction for the suggested approach is reduced $45 \%$ in contrast to the conventional FCS-MPC strategy. This means that the proposed control method provides the practical implementation of a real-time system with a high sampling rate and long prediction horizons.

The remaining of this paper is divided into four sections: The first section presents the mathematical model of DMC. A new methodology of the control strategy is outlined in the second section. A comparative examination of the traditional FCS-MPC and the proposed technique is analyzed with Matlab software in Sect. 3. Some conclusions are drawn in the final section.

\section{Model of direct matrix converter}

In this section, we consider a simple of a DMC as illustrated in Fig. 1. The DMC includes nine bidirectional switches which generate 27 possible switching states $[14,17]$. To avoid short 
circuit at the supply side and interrupted current flow at the load side, each output must be connected to only one input. The switching states of DMC are given by (1) [18]:

$S_{A a}+S_{A b}+S_{A C}=1$

$S_{B a}+S_{B b}+S_{B C}=1$,

$S_{C a}+S_{C b}+S_{C C}=1$,

where $S_{X Y}$ has two possible values: $\{0,1\}$ with the index $X \in\{A, B, C\}$ and $Y \in\{a, b, c\}$.

The model of the system can be described as follows:

$$
\begin{aligned}
u_{o}= & {\left[\begin{array}{lll}
S_{A a} & S_{A b} & S_{A C} \\
S_{B a} & S_{B b} & S_{B C} \\
S_{C a} & S_{C b} & S_{C c}
\end{array}\right] u_{e}=S u_{e^{\prime}} } \\
i_{e}= & {\left[\begin{array}{lll}
S_{A a} & S_{B a} & S_{C a} \\
S_{A b} & S_{B b} & S_{C b} \\
S_{A C} & S_{B C} & S_{C C}
\end{array}\right] i_{o}=S^{T} i_{o^{\prime}} }
\end{aligned}
$$

where $u_{o}=\left[\begin{array}{lll}u_{o a} & u_{o b} & u_{o c}\end{array}\right]^{T}, u_{e}=\left[\begin{array}{lll}u_{e a} & u_{e b} & u_{e c}\end{array}\right]^{T}$ are the load and input voltages; $i_{e}=\left[\begin{array}{lll}i_{e a} & i_{e b} & i_{e c}\end{array}\right]^{T}, i_{o}=\left[\begin{array}{lll}i_{o a} & i_{o b} & i_{o c}\end{array}\right]^{T}$ are the input and load currents.

The continuous-time model of the input side is performed as:

$$
\begin{aligned}
& \frac{d u_{e}}{d t}=\frac{1}{C_{f}} i_{s}-\frac{1}{C_{f}} i_{e} \\
& \frac{d i_{s}}{d t}=-\frac{1}{L_{f}} u_{e}-\frac{R_{f}}{L_{f}} i_{s}+\frac{1}{L_{f}} u_{s}
\end{aligned}
$$

where $u_{s}=\left[\begin{array}{lll}u_{s a} & u_{s b} & u_{s c}\end{array}\right]^{T}, i_{s}=\left[\begin{array}{lll}i_{s a} & i_{s b} & i_{s c}\end{array}\right]^{T}$ denote the source voltage and current. $R_{f}, L_{f}$, and $C_{f}$ stand for the resistance, inductance, and capacitance of the filter, respectively.

The behavior of load voltage and current is given by:

$\frac{d i_{o}}{d t}=\frac{1}{L}\left(u_{o}-R i_{o}\right)$,

where $R$ and $L$ represent the load resistance and inductance.

According to (3) and (4), the discrete-time dynamic model of the system is obtained by employing the forward Euler algorithm for a sampling interval of $T_{s}$ as follows:

$x(k+1)=A_{S(k)} x(k)+B u(k)$,

where

$$
\begin{aligned}
x(k) & =\left[\begin{array}{c}
u_{e}(k) \\
i_{s}(k) \\
i_{o}(k)
\end{array}\right], u(k)=u_{s}(k), \\
A_{S(k)} & =\left[\begin{array}{ccc}
1 & \frac{T_{s}}{C_{f}} & -S^{T}(k) \frac{T_{s}}{C_{f}} \\
-\frac{T_{s}}{L_{f}} & 1-\frac{T_{s} R_{f}}{L_{f}} & 0 \\
S(k) \frac{T_{s}}{L} & 0 & 1-\frac{T_{s} R}{L}
\end{array}\right], \\
B & =\left[\begin{array}{c}
0 \\
\frac{T_{s}}{L_{f}} \\
0
\end{array}\right]
\end{aligned}
$$

It is noted that at each sampling instant $k$, the switching state $S(k)$ is a switching combination which is limited to a bounded finite set $\mathbb{S}=\{1, \ldots, 27\}$ with each state $\in\{0,1\}$. Consequently, the system matrix $A_{S(k)}$ is finite-set constrained with the twenty-seven elements. The proposed method uses the discrete-time model to predict the load current. Then, the optimal switching state which is selected by minimizing the cost function will be implemented to the matrix converter.

\section{Proposed model predictive based on control Lyapunov function}

The major control goal of the proposed strategy is to achieve the load current tracking. Moreover, it can control the reactive power on the supply side to improve the source current. Nevertheless, this paper focuses on how to solve the closed-loop stability and high computational burden. Therefore, the further objective will not be developed here. A block diagram of the control system with a two-step prediction horizon illustrated in Fig. 2. With the aim to accomplish the control goal, the cost function of the proposed strategy is formed as the combination of the future and predicted load currents $[13,14,16]$ :

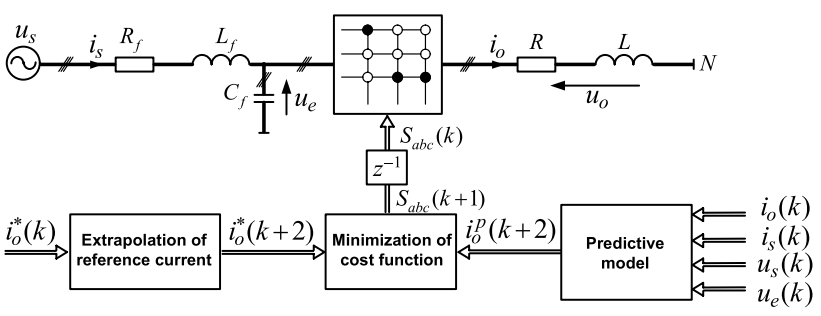

Fig. 2 Block diagram of the proposed FCS-MPC scheme with twostep prediction horizon 


$$
\begin{aligned}
g(S(k), S(k+1))= & \left\|i_{o}^{*}(k+2)-i_{o}^{p}(k+2)\right\|_{2}^{2} \\
& +\left\|i_{o}^{*}(k+1)-i_{o}^{p}(k+1)\right\|_{2^{\prime}}^{2}
\end{aligned}
$$

where $i_{o}^{*}(k+2)=\left[i_{o a}^{*}(k+2) i_{o b}^{*}(k+2) i_{o c}^{*}(k+2)\right]^{T}$, $i_{o}^{p}(k+2)=\left[i_{o a}^{p}(k+2) i_{o b}^{p}(k+2) i_{o c}^{p}(k+2)\right]^{T}$ indicate the load current references and predicted load currents at instant $k+2$, respectively.

The state errors of the load current are defined by:

$\widetilde{i}_{o}=i_{o}-i_{o^{\prime}}^{*}$

where $\widetilde{i}_{o}=\left[\begin{array}{lll}\tilde{i}_{o a} & \widetilde{i}_{o b} & \widetilde{i}_{o c}\end{array}\right]^{T}, i_{o}^{*}=\left[\begin{array}{lll}i_{o a}^{*} & i_{o b}^{*} & i_{o c}^{*}\end{array}\right]^{T}$.

By substituting (8) into (4), we have that:

$\frac{d \tilde{i}_{o}}{d t}=\frac{1}{L}\left(u_{o}-R \tilde{i}_{o}\right)-\frac{R}{L} i_{o}^{*}-\frac{d i_{o}^{*}}{d t}$

With the assumption that the sampling frequency $\left(f_{s}=50\right.$ $\mathrm{kHz})$ is greater than the frequency of the load current ( $f=50 \mathrm{~Hz}$ ), we can simplify as follows:

$\frac{d i_{o}^{*}}{d t}=0$.

The analysis of stability of the closed-loop system for indirect MPC strategy with a modulator is guaranteed by employing a Lyapunov function in the form of a terminal cost of the cost function. With these approaches, the optimization problem is formulated as a quadratic program or mixed-integer quadratic program is presented for hybrid systems such as DC boost converters [27, 28]. Concerning FCS-MPC, the topology of the power converters is considered as a linear system that is proved as practical stability [29]. However, this approach is not developed for complex converter topology. Another method based on the Lyapunov energy function is presented for voltage source converters [25, 26] and active filter [30]. These techniques employ negative Lyapunov function to guarantee the stability around the equilibrium point. In this study, a control Lyapunov function (CLF) is proposed to ensure the feasibility and stable closed-loop of the system:

$v\left(\widetilde{i}_{o a}, \widetilde{i}_{o b}, \widetilde{i}_{o c}\right)=\frac{1}{2} K_{a} \widetilde{i}_{o a}^{2}+\frac{1}{2} K_{b} \widetilde{i}_{o b}^{2}+\frac{1}{2} K_{c} \widetilde{i}_{o c}^{2}$

with derivative

$$
\begin{aligned}
\dot{v}\left(\widetilde{i}_{o a}, \widetilde{i}_{o b}, \widetilde{i}_{o c}\right)= & K_{a} \widetilde{i}_{o a} \frac{d \tilde{i}_{o a}}{d t}+K_{b} \widetilde{i}_{o b} \frac{d \tilde{i}_{o b}}{d t} \\
& +K_{c} \widetilde{i}_{o c} \frac{d \tilde{i}_{o c}}{d t},
\end{aligned}
$$

where $K_{a}, K_{b}, K_{c}$ stand for the positive gains.
The optimal solution of the proposed method based on the model predictive approach for two-step prediction is applied to the matrix converter at time $k+1$ as given:

$$
\begin{gathered}
S_{\text {opt }}=\arg \left\{\min _{S(k) \in \mathbb{S}, S(k+1) \in \mathbb{S}} g(S(k), S(k+1))\right\} . \\
\text { subject to } \quad \dot{V}(k)<0 \\
\dot{V}(k+1)<0
\end{gathered}
$$

The future reference load current is accomplished by utilizing the second-order Lagrange extrapolation as:

$i_{o}^{*}(k+2)=6 i_{o}^{*}(k)-8 i_{o}^{*}(k-1)+3 i_{o}^{*}(k-2)$.

As was discussed in Sect. 2, a compact set of 27 control switching signals must be evaluated for the optimization problem with one-step prediction. The control performance of the system can be improved by utilizing a long prediction horizon but increasing the computational burden due to the exhaustive search of the conventional FCSMPC technique. In this case, the computational complexity is increased exponentially along with control signal input and prediction horizon. As a result, this leads to cause a heavy computational load and restrict the practical horizon length of the controller for a given sampling period. In order to address this issue, we propose the Lyapunovbased MPC design which allows stabilizing the closed-loop system. In our study, a pruning approach is used for the selections inside the available finite set of control signals based on the decrease condition of the candidate Lyapunov function $V$. To reduce the candidate sequences of the control set, the function defined in (13) is employed for constructing the optimal cost search tree. The possible switching states only that fulfill the Lyapunov stability criteria at the prediction step $k+1$ are employed to evaluate the state variables required at the next sampling time $k+2$. Then, the search tree of the optimization is only conducted from reduced finite feasible sets with associated prediction step to select the best control input. As demonstrated in Fig. 3a, blue branches denote the available switching states, wherein the purple ones denote the infeasible solutions. In this case, at each prediction interval a candidate switching signal is satisfied a stability condition $(\dot{V}<0)$, then this available state is chosen for the evaluation of the optimization problem. It can be seen in Fig. 3b that several switching states are removed from the process of prediction and loop optimization due to the unsatisfactory stability condition. For example, at the time $t=0.0202 \mathrm{~s}$, there are only eleven switching states are determined as possible states selected for the optimization problem. Therefore, from the point of view of computational perspective, the proposed method can be improved by reducing the candidate switching state for 


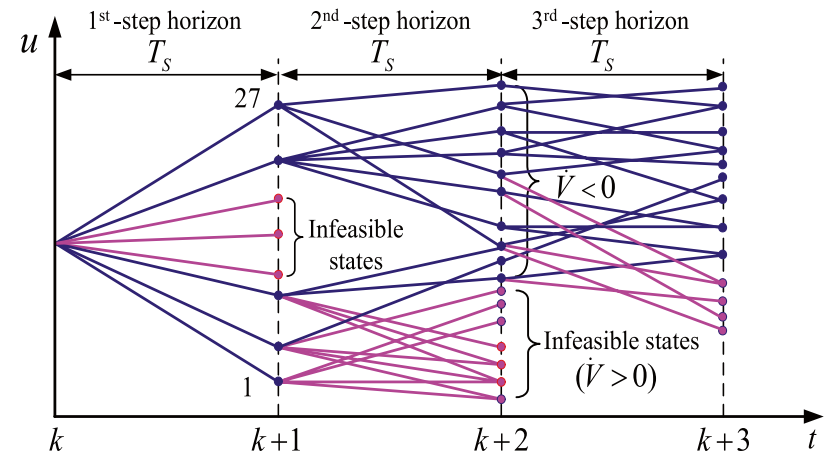

(a)

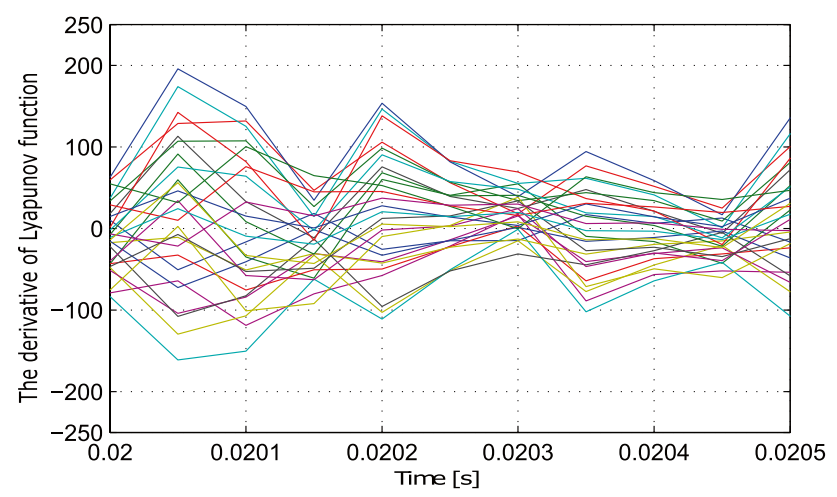

(b)

Fig. 3 The proposed algorithm with reduced candidate sequences: (a) The pruned search of candidate input sequences and (b) Zoom of the predictive behavior of time derivatives of a Lyapunov function for every switching signals

prediction and enumeration of the cost function instead of evaluating all considered switching states. Despite the unexpected increase of calculation due to the Lyapunov function, the computational burden of the proposed approach is dramatically decreased in contrast to the conventional method. In fact, the numbers of manipulated input of the conventional method for one-step and twostep predictions are 27 and 729, while their corresponding values of the proposed approach are 13 and 165, respectively. Table 1 shows the benefit of the proposed algorithm compared with the conventional method in respect of the decreased computational cost required, especially with

Table 1 The number of candidate control inputs reduced of optimization problem during sampling period concerning the prediction horizon length $(N)$

\begin{tabular}{llll}
\hline Prediction horizon & 1 & 2 & 3 \\
Conventional method & 27 & 729 & 19,683 \\
$\begin{array}{l}\text { Proposed technique } \\
\begin{array}{l}\text { Reduced control switching input } \\
\text { per sampling interval }\end{array}\end{array}$ & 13 & 165 & 4120 \\
\hline
\end{tabular}

the long prediction horizon. It is important to note that our technique provides a cutting-edge solution for the challenge of FCS-MPC for multilevel converters: closed-loop stability and heavy computational burden. Therefore, this strategy has the potential to be an efficient solution for implementing algorithms with low-cost processors. Finally, the proposed control algorithm is described as follows:

- Step 1 Measure $i_{o}(k), i_{s}(k), u_{s}(k)$ and $u_{e}(k)$. Read the reference load current $i_{o}^{*}(k)$.

- Step 2 Estimate the source current $i_{s}^{p}(k+1)$ by using (5).

- Step 3 Initialize optimal control switching signal and cost function.

- Step 4 Compute the prediction of input current $i_{e}^{p}(k+1)$ and voltage $u_{e}^{p}(k+1)$ from (2) and 5).

- Step 5 Calculate the derivative of the Lyapunov function for all possible states $\dot{V}_{k}^{i}$ with $i \in\{1, \ldots, 27\}$ from (9) and (12).

- Step 6 If $\dot{V}_{k}^{i}<0$ compute prediction of the load current $i_{o}^{p}(k+1)$ based on (5) and (6).

- Step 7 Calculate $\dot{V}_{k+1}^{j}$ with $j$ represents the predicted switching states at second step of prediction horizon $j \in\{1, \ldots, 27\}$ from (9) and (12).

- Step 8 If $\dot{V}_{k+1}^{j}<0$ predict $i_{o}^{p}(k+2)$ and evaluate the cost function $g\left(S_{k}, S_{k+1}\right)$ from (5) and (7). Minimize the cost function.

- Step 9 Apply optimal switching signal to the DMC.

\section{Results and discussions}

The effectiveness of the proposed control scheme is verified through simulation studies using the Matlab software. The parameters of the system appropriated for examination are given in Table 2.

In order to demonstrate the capability of the control strategy, several scenarios with different operating conditions are performed in this section. In the first scenario, a comparative study of the standard FCS-MPC and the proposed approach with the same conditions are analyzed.

Table 2 Parameters of the system for simulation validation

\begin{tabular}{lll}
\hline Parameter & Value & Description \\
\hline$U_{s}$ & $180[\mathrm{~V}]$ & Source phase voltage \\
$f$ & $50[\mathrm{~Hz}]$ & Frequency of source voltage \\
$R_{f}$ & $0.5[\mathrm{~m} \Omega]$ & Filter resistance \\
$L_{f}$ & $0.4[\mathrm{mH}]$ & Filter inductance \\
$C_{f}$ & $21[\mu \mathrm{F}]$ & Filter capacitance \\
$R$ & $10[\Omega]$ & Load resistance \\
$L$ & $30[\mathrm{mH}]$ & Load inductance \\
$T_{s}$ & $50[\mu \mathrm{s}]$ & Sampling time of controller
\end{tabular}


Fig. 4 The transient response and harmonic spectrum of the load current for the standard FCS-MPC, SMPC and proposed approaches

Moreover, the sequential model predictive control (SMPC) [31] is also compared to show the advantages of the proposed strategy. The root mean square (RMS) of the load current reference at $50 \mathrm{~Hz}$ changes from $8 \mathrm{~A}$ to $4 \mathrm{~A}$ at instant $t=0.065 \mathrm{~s}$. The dynamic response of three-phase load current for the proposed method is shown in Fig. 4a. As illustrated in Fig. 4b, the proposed technique obtains the similar performance of the load current compared with the standard FCS-MPC and SMPC. The rise time of the conventional method is $0.7 \mathrm{~ms}$, while the corresponding value of the proposed method and SMPC is $0.75 \mathrm{~ms}$. Specifically, three controllers have the same time to reach the steadystate of the reference current. The simulation result indicates that the proposed method achieves a capability of tracking reference current and fast transient performance. Moreover, a Powergui Fast Fourier Transform (FFT) toolbox is employed to evaluate the steady-state performance of the control system. The harmonic components of the load current for the standard, SMPC and the proposed method are also presented and analyzed in Fig. $4 d-f$. As shown in these figures, the total harmonic distortion of the current for the proposed approach increases inconsiderably from 2.16 to $2.32 \%$ in contrast with the standard FCS-MPC. Meanwhile, the increase of its value is $0.26 \%$ with respect to the SMPC. The small increase of THD is an unexpected result due to the incorporation of a stable closed-loop system. Nevertheless this, we can state that the performance of the system does not influence too much and still satisfy the IEEE standard 519 (5\%).

As reported previously, 729 predictions of the load current have to be evaluated in the optimization problem with the traditional FCS-MPC. On the contrary, only 165 average predictions are considered for the enumeration with the proposed method. Consequently, the computational cost of the proposed technique is significantly reduced in comparison to the standard FCS-MPC. Indeed, the average computation time of the conventional technique is about $70 \mu \mathrm{s}$ which is greater than the sampling interval $(50 \mu \mathrm{s})$. Meanwhile, the proposed algorithm requires just $38 \mu \mathrm{s}$ in a $2.5 \mathrm{GHz}$, i5-7200 CPU. Furthermore, the computation time of the proposed method is decreased by $33 \%$ compared with the SMPC strategy. This permit the feasibility of the real-time implementation with an increase in the prediction horizon. The strong impact of our contribution lies in the combination between the stability of the closed-loop system and reduced computational burden. The detail comparisons of three control methods which include the steady-state and dynamic performance are summarized in Table 3. The most remarkable result to emerge from the data is that the proposed methodology achieves the same

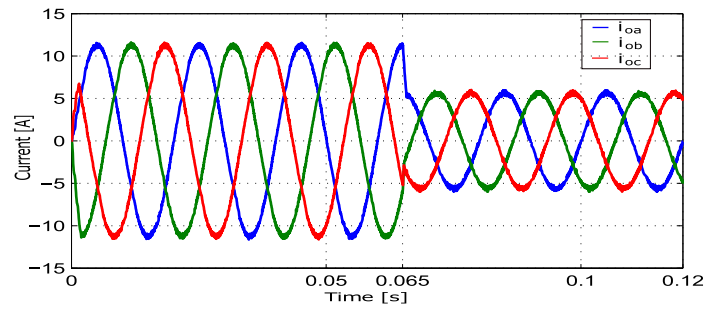

(a) Three-phase load current of the proposed method

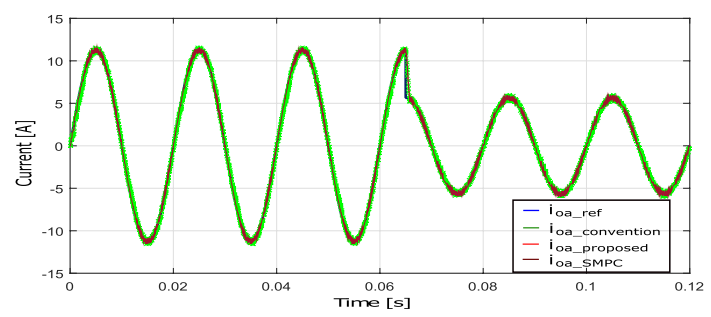

(b) Transient responses of the load current

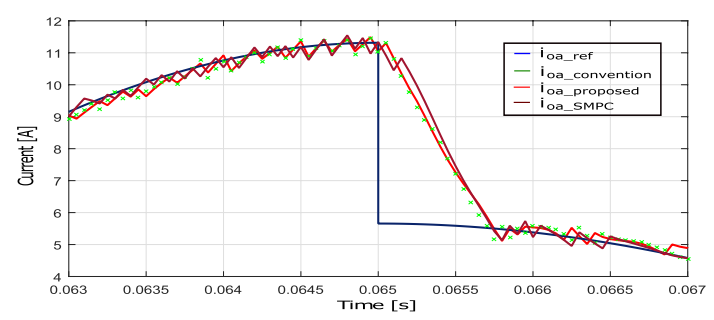

(c) Zoom of transient responses of the load current

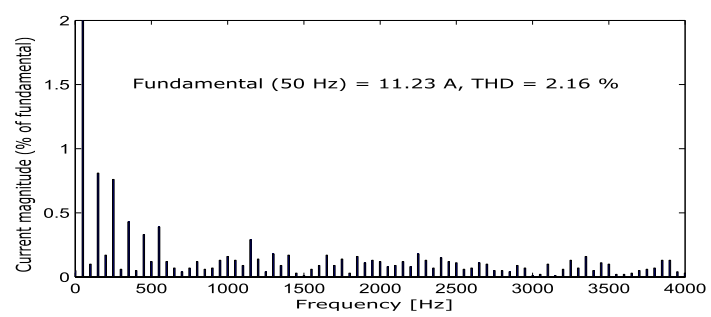

(d) FFT of the load current for the standard FCSMPC

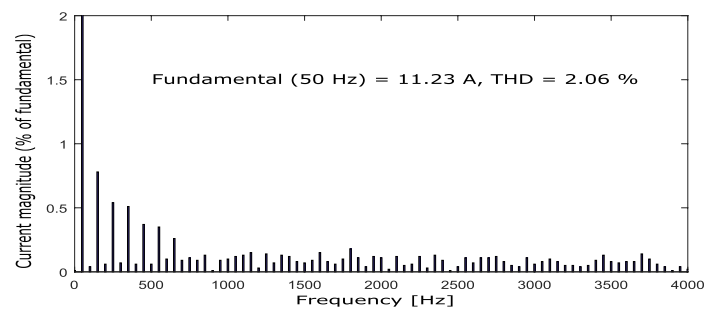

(e) FFT of the load current for the SMPC

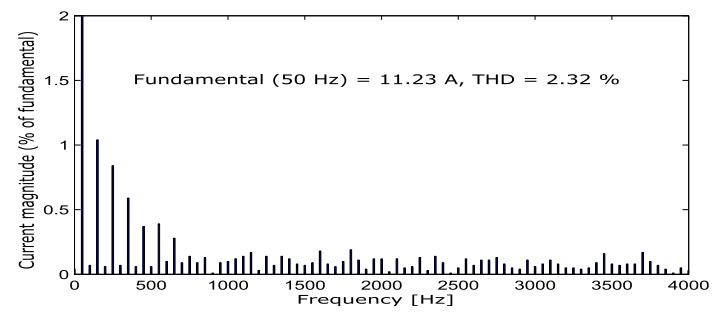

(f) FFT of the load current for the proposed method 
Table 3 Comparison of control performance for three control techniques with two-step prediction

\begin{tabular}{llll}
\hline Control performance & $\begin{array}{l}\text { Conven- } \\
\text { tional } \\
\text { method }\end{array}$ & SMPC & $\begin{array}{l}\text { Pro- } \\
\text { posed } \\
\text { method }\end{array}$ \\
\hline Rise time (ms) & 0.7 & 0.75 & 0.75 \\
Settling time (ms) & 0.85 & 0.85 & 0.85 \\
THD of load current (\%) & 2.16 & 2.06 & 2.32 \\
Average prediction states consid- & 729 & 596 & 165 \\
$\quad$ ered & & & \\
Minimum computation time $(\mu \mathrm{s})$ & 59 & 48 & 29 \\
Average computation time $(\mu \mathrm{s})$ & 70 & 57 & 38 \\
Maximum computation time $(\mu \mathrm{s})$ & 81 & 66 & 47 \\
Incorporation of stability criteria & No & No & Yes \\
\hline
\end{tabular}

performance in comparison to the conventional method and SPMC while solving the stability issue and high computational cost. Therefore, this study has the potential to apply the FCS-MPC for a multilevel converter with long prediction horizon and low-cost processor.

To examine the performance of the system under frequency variation, a step change in the load current reference from 50 to $30 \mathrm{~Hz}$ at $t=0.065 \mathrm{~s}$ is investigated in second scenarios. Figure 5 presents the dynamic response of the load current with the variation of the frequency. The proposed approach clearly accomplishes a good reference tracking despite the sudden change in the frequency.

To confirm the robustness of the controller under the unbalanced reference currents and unbalanced loads, the control scheme is also examined. We can chose the initial condition as the balanced reference currents $\left(I_{o a}=I_{o b}=I_{o c}=8 \mathrm{~A}\right)$ and unbalanced loads $\left(R_{a}=12 \Omega\right.$, $R_{b}=10 \Omega, R_{c}=8 \Omega$ ). Then, at instant $t=0.06 \mathrm{~s}$, the reference currents are set to $I_{o a}=5 \mathrm{~A}, I_{o b}=7 \mathrm{~A}$ and $I_{o c}=10 \mathrm{~A}$. It is apparent from Fig. 6 that the proposed technique is

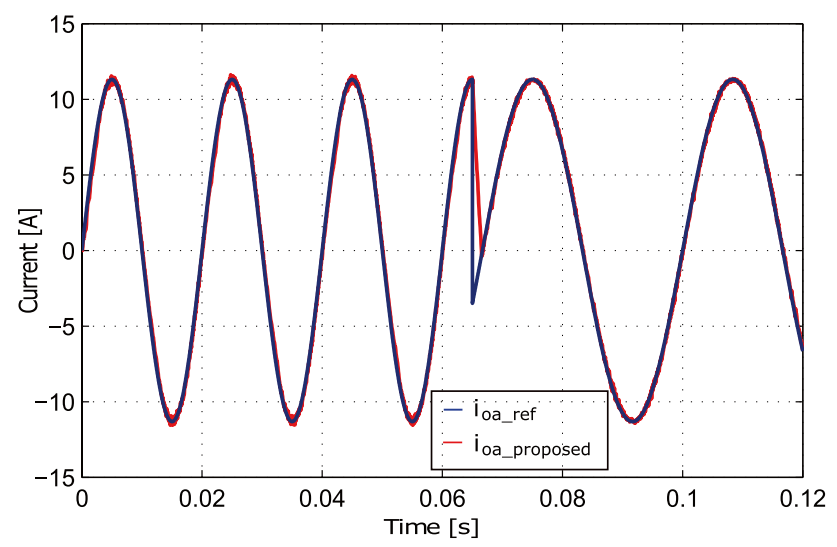

Fig. 5 The transient response of load current under dynamic change in frequency

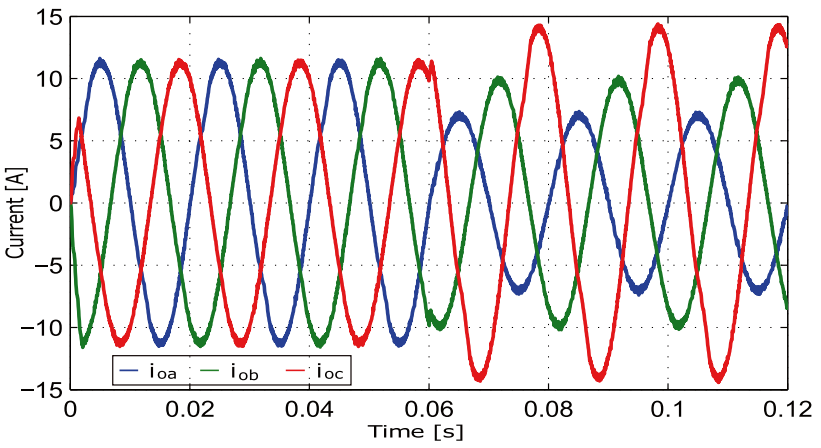

(a) Current response of three phases

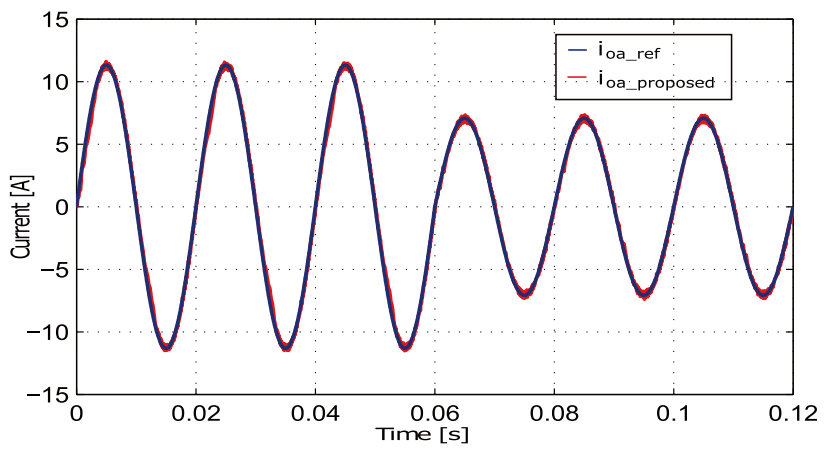

(b) Output load current and its reference

Fig. 6 Performance of the controller with unbalanced reference currents and unbalanced loads

capable to regulate the load current independently under the unbalanced conditions of the reference and load.

Another interesting aspect of the proposed control strategy is that it can keep the current tracking ability even if the supply voltage presents disturbances. To illustrate the performance of the control technique, the unbalanced source voltages are investigated. In this case, the RMS value of phase voltage $A$ is reduced $20 \%$ nominal value, while phase voltage $B$ is increased by $30 \%$ of its value, respectively as shown in Fig. 7a. According to Fig. $7 b$, the predictive method is continued to satisfy the control objective in spite of disturbances.

To validate the capability of the proposed technique deal with parameter variations, the load resistance and inductance have been increased to $50 \%$ of their real values and decreased to $50 \%$ at instant $t=0.06 \mathrm{~s}$, respectively. Figure 8 demonstrates that the proposed method can track its reference value with a moderate increase of the current ripple, but keeps a satisfactory performance. Hence, it is possible to indicate that the proposed control strategy is robust and can operate under the parameter variations.

A laboratory prototype was constructed to confirm the effectiveness of the proposed control scheme. The control algorithm was implemented in a digital signal 


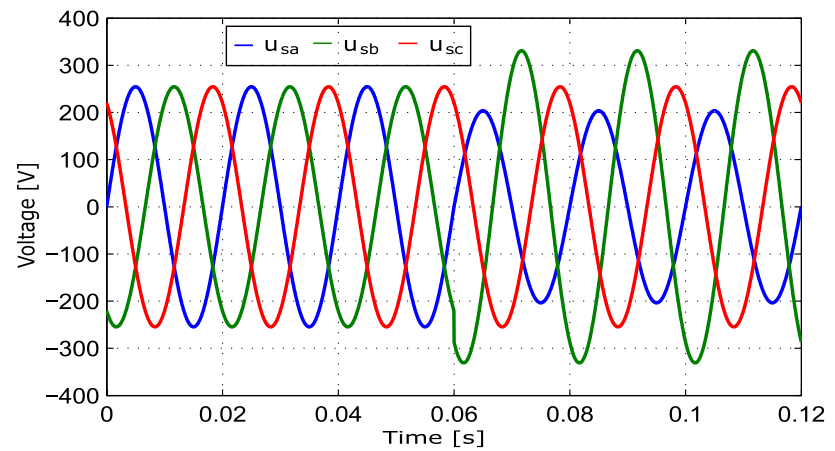

(a)

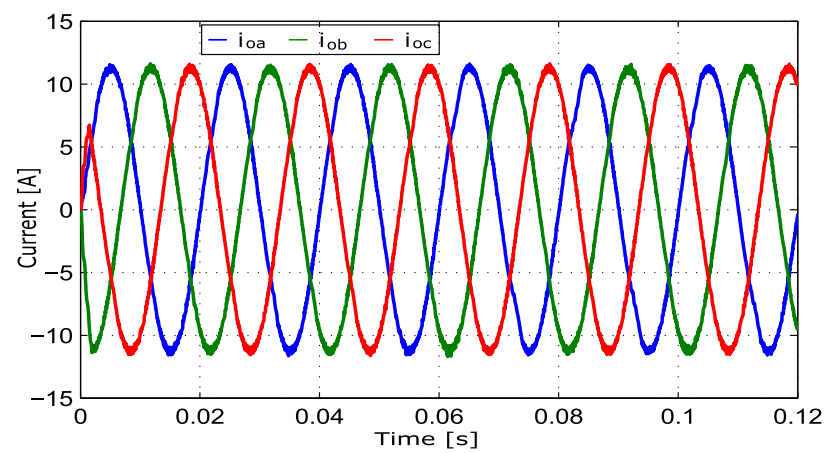

(b)

Fig. 7 Simulation results of the system under unbalanced supply voltage: (a) Source voltage of three-phases and (b) Waveform load current of three phases

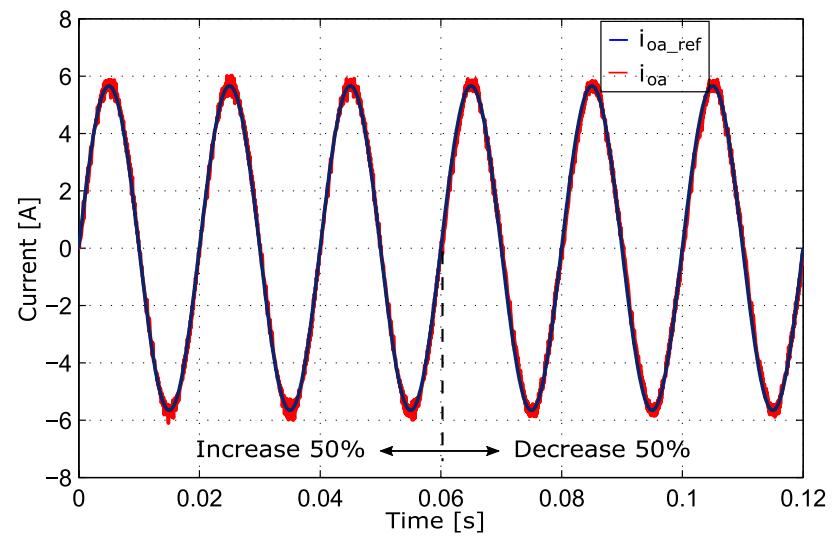

Fig. 8 Dynamic response of load current with parameter variations

processor TMS320F28335. The DMC was actualized with eighteen modules IGBT IRG4PF50WD. The LV 25-P and LA 25-P sensors were utilized to measure the currents and voltages. The root means square of the source phase voltage was fixed at $220 \mathrm{~V}$. The parameters of the system are the same as the simulation. In the first examination, the peak value of the load current is set at $8 \mathrm{~A}$ to analyze the steady-state performance of the control system.

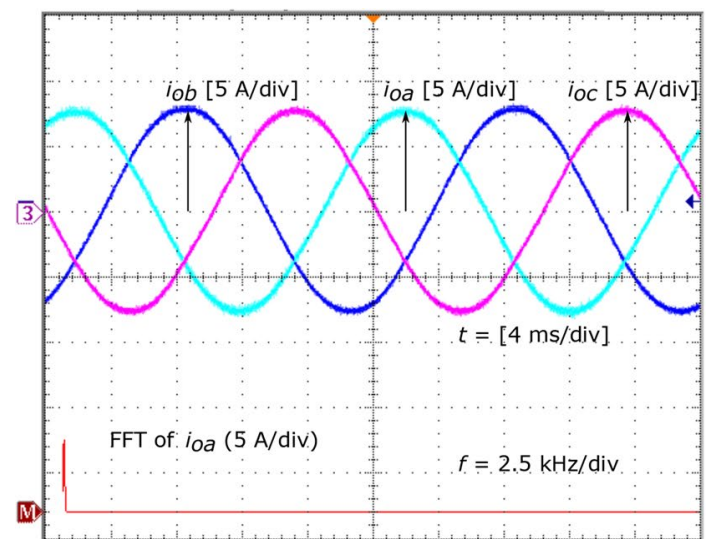

(a)

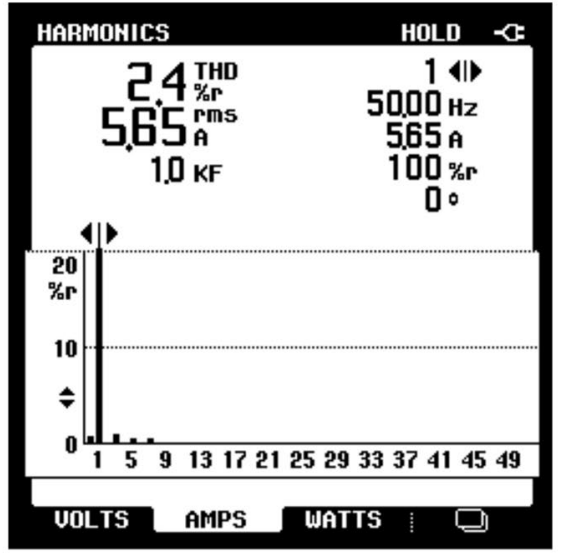

(b)

Fig. 9 Experimental waveforms of output load current: (a) Steadystate of three-phase current and (b) Harmonic spectrum of the current

Figure 9a illustrates the waveform of the three-phase output current of the DMC. From this figure, we can note that the proposed method achieves a good performance of output current. Moreover, as highlighted in Fig. 9b, the quality of the load current is analyzed throughout the total harmonic distortion that is suitable for the IEEE 516 standard (5\%). Finally, a further test was conducted under the transient operation of the system to validate the effectiveness of the proposed control strategy. The reference peak value of the current is changed from $8 \mathrm{~A}$ to $4 \mathrm{~A}$. As shown in Fig. 10, the output current reached the steady-state condition after a quick transient time. It is evident that the proposed technique exhibits good control performance during transient and steady-state operations. Therefore, our strategy is a clear improvement on current methods in terms of reducing the computational burden while preserving high control performance. 


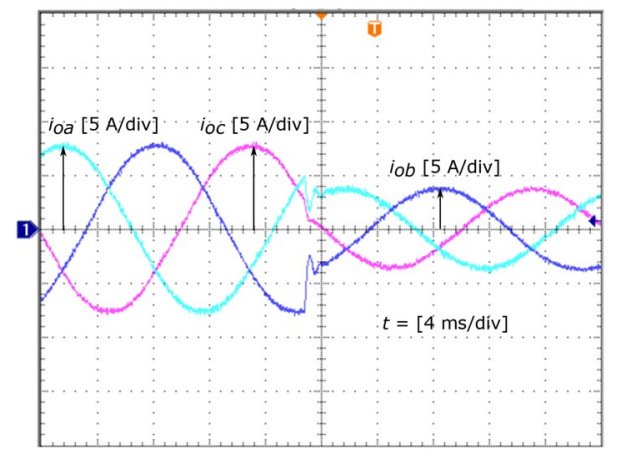

Fig. 10 Dynamic response of output load current

\section{Conclusion}

This paper introduced a powerful model predictive control strategy for DMC. The aim of the proposed approach is the development of FCS-MPC based on control Lyapunov function to address the challenges of applying FCS-MPC for DMC. In contrast to exhausted-search of conventional FCS-MPC, a pruning direct search based on the predicted immediate stability of each future control input is taken into account in the optimization problem. Therefore, we accomplished a notable reduction of computation time in comparison to the conventional FCS-MPC, resulting in facilitating the real-time implementation of the MPC method. A comparative analysis of the proposed approach, conventional FCS-MPC and SMPC is examined to illustrate the effectiveness of the control scheme in respect of computational burden, stability analysis, and control performance. The simulation and experimental results confirm the feasibility of the proposed technique to apply for DMC with long-horizon prediction. We believe that our results may improve knowledge about using stability to reduce the computational load for other converter configuration.

Acknowledgements This research was supported by Research Foundation funded by Thai Nguyen University of Technology.

\section{Compliance with ethical standards}

Conflict of interest The authors declare that they have no conflict of interest.

\section{References}

1. Empringham L, Kolar JW, Rodriguez J, Wheeler PW, Clare JC (2013) Technological issues and industrial application of matrix converters: a review. IEEE Trans Ind Electr 60(10):4260-4271

2. Wheeler PW, Rodriguez J, Clare JC, Empringham L, Weinstein A (2002) Matrix converters: a technology review. IEEE Trans Ind Electr 49(2):276-288
3. Casadei D, Serra G, Tani A (2001) The use of matrix converters in direct torque control of induction machines. IEEE Trans Ind Electr 48(6):1057-1064

4. Vargas R, Rodriguez J, Ammann U, Wheeler PW (2008) Predictive current control of an induction machine fed by a matrix converter with reactive power control. IEEE Trans Ind Electr 55(12):4362-4371

5. Mondal S, Kastha D (2015) Improved direct torque and reactive power control of a matrix-converter-fed grid-connected doubly fed induction generator. IEEE Trans Ind Electr 62(12):7590-7598

6. Jianwei Z, David GD, Li L, Youguang G (2018) Decoupling controller design and controllable regions analysis for the space vector modulated matrix converter unified power flow controller in transmission systems. Electr Power Compon Syst 46:1-14

7. Alesina A, Venturini MGB (1989) Analysis and design of optimum-amplitude nine-switch direct AC-AC converters. IEEE Trans Power Electr 4(1):101-112

8. Idris Z, Hamzah MK, Omar AM (2005) Implementation of singlephase matrix converter as a direct AC-AC converter synthesized using sinusoidal pulse width modulation with passive load condition. PEDS 2:1536-1541

9. Casadei D, Serra G, Tani A, Zarri L (2002) Matrix converter modulation strategies: a new general approach based on spacevector representation of the switch state. IEEE Trans Ind Electr 49(2):370-381

10. Hamouda M, Fnaiech F, Al-Haddad K, Kanaan HY (2004) Matrix converter control: a sliding mode approach. IECON 3:2295-2300

11. Pinto S, Silva J (2007) Sliding mode direct control of matrix converters. IET Electr Power Appl 1(3):439-448

12. Ortega C, Arias A, Espina J (2004) Predictive direct torque control of matrix converter fed permanent magnet synchronous machines. Asian J Control 16(1):70-79

13. Rivera M, Wilson A et al (2013) A comparative assessment of model predictive current control and space vector modulation in a direct matrix converter. IEEE Trans Ind Electr 60(2):578-588

14. Rodriguez J, Cortes P (2012) Predictive control of power converters and electrical drives. John Wiley, New Jersey

15. Ngo VQB, Nguyen MK, Tran TT, Lim YC, Choi JH (2019) A modified model predictive power control for grid-connected T-type inverter with reduced computational complexity. Electronics $8(2): 217$

16. Rodriguez J, Kazmierkowski MP, Espinoza JR, Zanchetta P, AbuRub H et al (2013) State of the art of finite control set model predictive control in power electronics. IEEE Trans Ind Inform 9(2):1003-1016

17. Vijayagopal $M$, Zanchetta $P$, Empringham L, de-Lillo L, Tarisciotti $L$ et al (2017) Control of a direct matrix converter with modulated model predictive control. IEEE Trans Ind Appl 53(3):2342-2349

18. Dan H, Zhu Q, Peng T, Yao S, Wheeler P (2017) Preselection algorithm based on predictive control for direct matrix converter. IET Electr Power Appl 11(5):768-775

19. Gulbudak O, Santi E (2016) FPGA-based model predictive controller for direct matrix converter. IEEE Trans Ind Electr 63(7):4560-4570

20. Siami M, Khaburi DA, Rodriguez J (2018) Simplified finite control set-model predictive control for matrix converter-fed PMSM drives. IEEE Trans Power Electr 33(3):2438-2446

21. Marcucci T, Russ T (2020) Warm start of mixed-integer programs for model predictive control of hybrid systems. IEEE Trans Autom Control. https://doi.org/10.1109/TAC.2020.3007688

22. Fletcher $R$, Sven $L$ (1998) Numerical experience with lower bounds for MIQP branch-and-bound. SIAM J Optim 8(2):604-616

23. Bartolomeo S, Vihangkumar VN, Alberto B, Paul G, Stephen B (2018) Embedded mixed-integer quadratic optimization using the OSQP solver. In: ECC, pp 1536-1541 
24. Karamanakos P, Liegmann E, Geyer T, Kennel R (2020) Model predictive control of power electronic systems: methods, results, and challenges. IEEE Open J Ind Appl 1:95-114

25. Kwak S, Yoo SJ, Parka J (2014) Finite control set predictive control based on Lyapunov function for three-phase voltage source inverters. IET Power Electr 7(11):2726-2732

26. Akter MP, Mekhilef S, Tan NML, Akagi H (2016) Modified model predictive control of a bidirectional AC-DC converter based on Lyapunov function for energy storage systems. IEEE Trans Ind Electr 63(2):704-715

27. Bemporad A, Heemels WPMH, Schutter BD (2002) On hybrid systems and closed-loop MPC systems. IEEE Trans Autom Control 47(5):863-869

28. Geyer T, Papafotiou G, Morari M (2008) Hybrid model predictive control of the step-down dc-dc converter. IEEE Trans Control Syst Technol 16(6):1112-1124
29. Aguilera RP, Quevedo DE (2013) Stability analysis of quadratic MPC with a discrete input alphabet. IEEE Trans Autom Control 58(12):3190-3196

30. Rahmani S, Hamadi A, Al-Haddad K (2012) A Lyapunov-functionbased control for a three-phase shunt hybrid active filter. IEEE Trans Ind Electr 59(3):1418-1429

31. Jianwei Z, Margarita N, Li L, David D, Rodriguez J (2019) Sequential model predictive control of three-phase direct matrix converter. Energies 12(2):214

Publisher's Note Springer Nature remains neutral with regard to jurisdictional claims in published maps and institutional affiliations. 